EDITORIAL

\title{
National variations in cardiac service provision: how united is our kingdom?
}

\author{
M S Norell, K P Jennings
}

\section{Has devolution in the UK brought about a disparity in cardiological resources, putting those citizens in the devolved countries at a disadvantage?}

Heart 2006;92:1-2. doi: 10.1136/hrt.2005.070292

the complexities of attempting to analyse these variations. How does one take account of consultant cardiologists or cardiac surgeons working whole time or part time in their specialty? Should activity undertaken in private institutions be included? How is cross border activity to be analysed in order to avoid double counting? To address these issues a number of assumptions were made and clearly outlined in the report.

\section{DISCREPANCIES}

The messages that emerge are clear and consistent. A greater burden of CAD, as measured by age standardised mortality, is apparent in the devolved nations in comparison with England. Despite this, Wales and Scotland fair less well than England or Northern Ireland, when measures of service provision are analysed. The number of catheter laboratories per head of the population is smaller in Scotland and Wales, which predictably perform fewer diagnostic angiograms with respect to the size of their populations. Waiting time targets, which also measure the aspirations of a service, are shown to range considerably. That for outpatient consultation varies from 13 weeks in England to a remarkable 18 months in Wales. ${ }^{3}$

Revascularisation rates also vary across nations with Wales performing the lowest number of PCI procedures. Cardiac surgical activity, as indicated by the number of CABG operations undertaken, is also lower in Wales in comparison with England and Scotland. Conversely, Northern Ireland performs proportionately least CABG procedures but the greatest volume of PCI. This is likely to relate to their having the lowest number of cardiac surgeons in post but the largest number of cardiologists. These differences also extend to the implementation of new technologies; drug eluting stent usage and new AICD implantation rates are lower in Scotland and Wales in comparison with England and Northern Ireland.

Perhaps of more concern is that these variations may not simply relate to resource availability. The application of services, and the mechanisms by which nationally generated recommendations are implemented, are not the same across the UK. The report describes each of

Abbreviations: $A I C D$, automatic implantable cardioverter-defibrillator; $C A B G$, coronary artery bypass grafting; CAD, coronary artery disease; MINAP, Myocardial Infarction Audit Project; NICE, National Institute for Health and Clinical Excellence; NSF, National Service Framework; $\mathrm{PCl}$, percutaneous coronary intervention

While such comparisons may appear straightforward at first glance, the report also illustrates eluting stents and the implantation rates of atic implantable cardioverter-defibrill (AICDs). Importantly, the report was underpinned by a comparison of the burden that CAD 
these processes and demonstrates their differences. So for example, guidance from NICE, which will have incorporated specialist contributions from all four countries, may still be reappraised before implementation in Scotland.

\section{CONCERN}

The report makes stark reading and confirms the perception of many observers that discrepancies exist. As the British Cardiac Society represents cardiological interests across the whole of the UK, such findings are seen as unacceptable. It urges that when specialist societies and national agencies produce guidelines and recommendations, these are accepted in all devolved nations without the need for further modification. Similarly if European guidelines are embraced by UK organisations, these should also be acknowledged across all our nations, and implemented in an unaltered form. Individual assemblies and parliaments need to address the deficiencies that are apparent to ensure equal access to cardiac services across all of the UK.

The release of the report to ministers and national societies will no doubt prompt an early response, but we must remain vigilant. We need to ensure that implementation and funding of agreed guidelines and recommendations, such as those from NICE, are mandated in the devolved nations as well as in England. Lobbying of ministers and government agenciesperhaps through the media-should exert sustained pressure.
This review should not necessarily be seen as a damning indictment of differing cardiac services. Rather than represent simply a criticism of specific governments or assemblies, it should instead be welcomed as a catalyst for further joint discussion and action. Working with ministers and government agencies, we must continue to highlight persisting differences in national practice and maintain the focus of health departments upon areas of concern. Only in this way will all the citizens of the UK benefit equally from the many advances available to our patients with CAD. In order to monitor any progress a repeat review is planned next year.

\section{Authors' affiliations}

M S Norell, Department of Cardiology, The Heart and Lung Centre, Wolverhampton, UK

K P Jennings, Department of Cardiology, Royal Infirmary, Aberdeen, UK

\section{REFERENCES}

1 Department of Health. The National Service Framework for coronary artery disease. London: Department of Health, 2000.

2 Brooks N, Norell M, Holl J, et al. National variations in the provision cardiac services in the United Kingdom. A report by a working group of the British Cardiac Society. Br J Cardiol 2005;12:192-8.

3 Welsh Assembly Government. Welsh Health Circular WHC (2003) 127 Annual priorities and planning guidance for the Service and Financial Framework 2004-5, Welsh Assembly Government, 19 December, 2003.

\title{
IMAGES IN CARDIOLOGY
}

Multislice cardiac computed tomographic images of anomalous origin of the left coronary artery from the pulmonary artery (ALCAPA)

\begin{abstract}
A 54 year old man with no modifiable coronary risk factors presented with a 10 year history of insidious onset of progressive exertional dyspnoea. He denied history of paroxysmal nocturnal dyspnoea, orthopnoea, palpitations, syncope, oedema, or claudication. Clinical examination revealed a mesomorphic normotensive male with atrial fibrillation (AF), cardiomegaly, moderate mitral regurgitation (MR), mild tricuspid regurgitation (TR), and pulmonary arterial hypertension (PAH). A chest $x$ ray (posterioanterior) view revealed cardiomegaly, while his ECG revealed left bundle branch block and AF. Echo Doppler evaluation revealed a dilated, globally hypokinetic left ventricle with an ejection fraction of $45 \%$, moderate MR, PAH, and mild TR.

Multislice cardiac computed tomography (16 slices) revealed anomalous origin of the left coronary artery from the pulmonary artery (ALCAPA) receiving collaterals from a normally originating dilated tortuous right coronary artery (panels). There were specks of coronary calcification, but no stenotic lesions. Coronary angiography confirmed the diagnosis and the patient underwent successful coronary artery bypass graft (CABG) surgery after closure of the ostium (in the main pulmonary artery) of the anomalously originating left coronary artery. Multislice cardiac computed tomography is thus a useful modality for diagnosing congenital coronary anomalies.
\end{abstract}
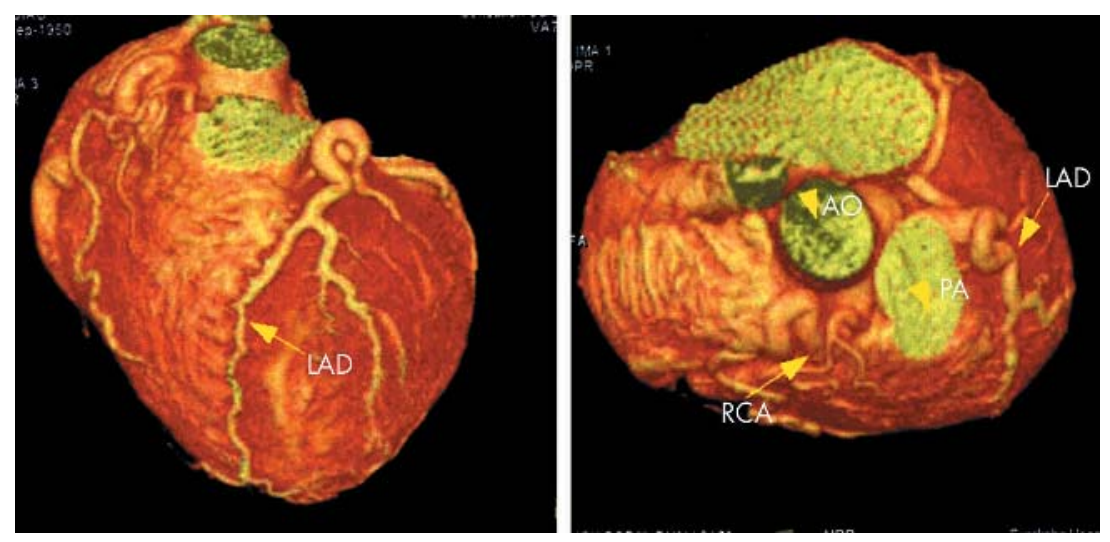

R Girish

gir63@yahoo.com 